\title{
Prognostic implications of pathogenic truncating variants in the TTN gene
}

\author{
Maria Luisa Peña-Peña ${ }^{\mathrm{a}}$, Juan Pablo Ochoa $^{\mathrm{b}}$, Roberto Barriales-Villa ${ }^{\mathrm{c}, \mathrm{d}}$, Marcos \\ Cicerchia $^{\mathrm{b}}$, Julián Palomino-Doza ${ }^{\mathrm{d}, \mathrm{e}}$, Joel Salazar-Mediguchia ${ }^{\mathrm{b}}$, Arsonval \\ Lamounier $^{\mathrm{b}}$, Juan Pablo Trujillo ${ }^{\mathrm{b}}$, Diego Garcia-Giustiniani ${ }^{\mathrm{b}}$, Xusto Fernandez ${ }^{\mathrm{b}}$, \\ Martin Ortiz-Genga ${ }^{\mathrm{b}}$, Lorenzo Monserrat ${ }^{\mathrm{b}}$, Maria Generosa Crespo-Leiro ${ }^{\mathrm{c}, \mathrm{d}}$. \\ ${ }^{a}$ Cardiology Department, Virgen del Rocio University Hospital, Seville, Spain, A Coruña University (UDC), $\quad$ A Coruña \\ Spain \\ ${ }^{b}$ Institute of Biomedical Research of A Coruña (INIBIC), Clinical Department, Health in Code, A Coruña, Spain \\ ${ }^{c}$ Cardiology Department, Complexo Hospitalario Universitario A Coruña (CHUAC), A Coruña University (UDC), INIBIC, \\ A Coruña, Spain \\ ${ }^{d}$ Centro de Investigación Biomédica en Red de Enfermedades Cardiovasculares (CIBERCV), Instituto de Salud Carlos III \\ Madrid, Spain \\ ${ }^{e}$ Cardiology Department, 12 de Octubre University Hospital, Madrid, Spain
}

\begin{abstract}
Introduction and objectives

TTN gene truncating variants (TTNtv) are a frequent cause of dilated cardiomyopathy (DCM). However, there are discrepant data on the associated prognosis. Our objectives were to describe the prevalence of TTNtv in our cohort and to compare the clinical course with that described in the literature.

Methods

We included patients with DCM and genetic testing performed using next-generation sequencing. Through a systematic literature research, we collected information about carriers and affected relatives with TTNtv. We compared the cumulative percentage of affected carriers and the survival free of cardiovascular death.

Results

One hundred and ten DCM patients were evaluated. A total of 13 TTNtv distributed in 14 probands were identified (12.7\%). We found a $21.4 \%$ prevalence in familial cases. No significant differences in the relation between age and clinical disease expression were identified. Survival free of cardiovascular death curves constructed from data in the literature seems not to overestimate the risk in our population.

Conclusions

The identification of TTNtv in patients with DCM is frequent and provides relevant information about the disease prognosis. The risk of cardiovascular death should not be underestimated. Age related penetrance need to be considered in the familial evaluation.
\end{abstract}

Keywords: Dilated cardiomyopathy; Genetic testing; Mutation

Abbreviations: DCM. dilated cardiomyopathy; TTNtv, titin gene truncating variants; NGS, next-generation sequencing 


\section{Introduction}

Titin gene truncating variants (TTNtv) are a frequent cause of dilated cardiomyopathy (DCM), occurring in $14-25 \%$ of cases $[\underline{1}, 2]$. Described penetrance of pathogenic TTNtv is age-dependent and varies among different cohorts [[2], [3], [4]]. There are limited and discrepant data in the literature on the associated prognosis. Case series have reported the highest prevalence of TTNtv among patients with severe disease $[2, \underline{5}]$. However, in a clinical study of DCM probands and relatives, a milder course than in carriers of mutations in $L M N A$ or negative genetic testing was observed []] .

Our objectives were to describe the prevalence of TTNtv in a cohort of DCM patients and to compare the clinical course with that described in the literature for TTNtv carriers.

\section{Methods}

\section{Patient cohort}

From February 2014 to December 2018, 110 patients with DCM from A Coruña University Hospital were studied after informed consent with next-generation sequencing (NGS) panels including TTN and at least 80 other candidate genes. Clinical and genetic familial screenings were performed. The diagnosis of DCM was done following the European Society of Cardiology criteria [6]. DCM was considered familial when two or more individuals were affected, or in the presence of sudden unexplained death in a first-degree relative at $<45$ years of age [7].

The study protocol was approved by the Research Ethics Committee of A Coruña-Ferrol and followed the Helsinki declaration recommendations.

\section{Patients from literature}

We performed a comprehensive bibliographic search of studies published in PubMed (1 January 1971 to Dec 2018) to collect available clinical information of families and individuals who carried TTNtv associated with DCM. The following search terms were used: "English"[Language] AND (“1900/01/01"[Date - Publication]: “2018/12/31" [Date - Publication]) AND (“dilated cardiomyopathy" OR “cardiomyopathy") AND ("titin" OR "TTN").

The search was run on 10 of January of 2019 and retrieved 328 citations. All titles and abstracts of the articles were evaluated by two independent reviewers (L.M. and J.P.O.). After exclusion based on the title and abstract, a first selection of candidate articles was made. The full text of these articles was evaluated. We included affected and unaffected individuals following the same criteria used for our cases.

\section{Genetic studies}

Coding exons and intronic boundaries of at least 80 genes related to DCM were sequenced using the HiSeq 1500 platform (Illumina, San Diego, California, USA) following lllumina protocols. TTN $v$ were defined as those that were predicted to introduce a premature stop codon in the protein's sequence (nonsense or frameshift) or that could alter the splicing process according to the predictions of five software tools: MaxEntScan, Splice-Site Finder, HSF, NNSPLICE, and GeneSplicer, in any of the described protein isoforms. Pathogenicity of variants was classified according to current recommendations []]. 
Cardiovascular death was defined as the presence of sudden cardiac death, appropriate defibrillator shock, heart failure death, heart transplantation, death related to a cardiovascular intervention or stroke related death. For survival analysis we only considered carriers with pathogenic or likely pathogenic variants and available data. Survival free of cardiovascular death was estimated using the Kaplan-Meier method; factors were compared using the log-rank (MantelCox) test. Survival was calculated from birth. A two-sided $p$ value $<0.05$ was considered to indicate statistical significance. Analysis was performed using the R version 3.4.3 (The R Foundation for Statistical Computing Platform).

\section{Results}

\section{Molecular findings}

A total of 13 TTNtv distributed in 14 probands were identified (Table 1), representing 12.7\% of the 110 consecutive DCM patients who were sequenced. Nine of 42 familial cases $(21.4 \%)$ carried a TTN $t v$ compared to 5 of 68 sporadic patients $(7.4 \%)$. The variant p.Glu3810* was identified in 2 unrelated probands. A total of 61 probands' relatives were identified. After informed consent and clinical study, targeted genetic testing was performed in 33 family members. Among relatives, 18 mutations carriers were identified: 5 were clinically affected $(27.8 \%)$ and $13(72.2 \%)$ were considered not affected at a median age of $39.4 \pm 19.3$ years. Fifteen relatives were non-carriers. 
Table 1. Description of the identified TTN truncation variants in our cohort.

\begin{tabular}{|c|c|c|c|c|c|c|c|c|}
\hline Refseq transcript & Protein (NP_003310.4) & Chromosomic (NC_000002.11) & Localization & $\begin{array}{l}\text { Exon } \\
\text { number }\end{array}$ & Exon PSI $\%$ & GnomAD MAF\% & Family identifier & $\begin{array}{l}\text { Carriers } \\
\text { (affected/unaffected) }\end{array}$ \\
\hline NM_00331 9.4 & p.Thr300Aspfs $* 23$ & g.179664231_179664232insA & $\mathrm{Z}$ disk & 6 & 100 & No & 41,958 & $1(1 / 0)$ \\
\hline NM_00331 9.4 & p.Glu3810* & g. $179605443 \mathrm{C}>\mathrm{A}$ & I band & 45 & 100 & No & $36,814,3567$ & $5(3 / 2)$ \\
\hline NM_00331 9.4 & p.Asn4176* & g.179604349_179604350insA & I band & 45 & 100 & No & 36,815 & $2(1 / 1)$ \\
\hline NM_00331 9.4 & p.Arg4375* & g. $179602968 \mathrm{G}>\mathrm{A}$ & I band & 46 & 100 & No & 25,921 & $2(1 / 1)$ \\
\hline NM_00331 9.4 & p.Arg8272* & g. $179474028 \mathrm{G}>\mathrm{A}$ & A band & 101 & 100 & No & 41,933 & $3(2 / 1)$ \\
\hline NM_00331 9.4 & p.Tyr9697* & g. $179464342 \mathrm{~A}>\mathrm{C}$ & A band & 117 & 100 & No & 41,871 & $4(2 / 2)$ \\
\hline NM_00331 9.4 & p.Trp13407* & g. $179444509 \mathrm{C}>\mathrm{T}$ & A band & 147 & 100 & No & 41,930 & $3(2 / 1)$ \\
\hline NM_00331 9.4 & p.Val16403Glufs*33 & g.179434456delA & A band & 154 & 100 & No & 41,937 & $2(1 / 1)$ \\
\hline NM_003319.4 & p.Lys16797Argfs*25 & g.179433275delT & A band & 154 & 100 & No & 41,932 & $4(2 / 2)$ \\
\hline NM_00331 9.4 & p.Tyr $17457^{*}$ & g. $179431293 \mathrm{~A}>\mathrm{C}$ & A band & 154 & 100 & $<0.001 \%$ & 41,869 & $3(1 / 2)$ \\
\hline NM_00331 9.4 & p.Arg17841* & g. $179430143 \mathrm{G}>\mathrm{A}$ & A band & 154 & 100 & $<0.001 \%$ & 33,818 & $1(1 / 0)$ \\
\hline NM_00331 9.4 & p. Pro $18608 \mathrm{Glnfs} * 3$ & g.179427842_179427843delGTinsA & A band & 154 & 100 & No & 41,925 & $1(1 / 0)$ \\
\hline NM_00331 9.4 & p.Ala12453Serfs*14 & :g.179449922_179449923insT & A band & 137 & 100 & No & 51,338 & $1(1 / 0)$ \\
\hline
\end{tabular}




\section{Clinical characteristics of patients carrying TTNtv}

Seventy-five percent of the probands were male. Age at clinical diagnosis of the disease was highly variable both in our cohort (range from 16 to 69 years) and in the literature (range from 1 to 78 ). The median age of diagnosis was 52 years old in our cohort and 43 in carriers from the literature $(\mathrm{p}=0.13)$. The maximum incidence of clinical diagnosis was observed between ages 40 and 60 (Fig. 1A).

No significant differences in survival free of cardiovascular death, transplant or appropriate ICD discharge were observed between our cases with TTNtv and those from the bibliography $(\mathrm{p}=0.17)$, although there was a trend toward a worse prognosis in our patients at advanced ages (Fig. 1B).

In our cohort, 8 patients belonging to different families required a heart transplant. There were 2 heart failure deaths in relatives with DCM and sudden cardiac death occurred in 4 patients belonging to the group of 51-60 years. In the group of carriers identified in the literature, heart failure deaths and transplants also predominated, but sudden cardiac death was frequent especially in younger patients $(<40$ years old $)$.

\section{Discussion}

While studying 110 DCM patients we identified 14 TTNtv, 13 of them novel. The observed frequency is similar as in other published cohorts $[2,5]$. The majority of variants were located in the A-band region and all affected highly expressed exons, consistent with the findings published so far [5]. Penetrance increases with age, with a peak of incidence of new diagnosis between 40 and 60 years. This stress the importance of a clinical follow-up until advances ages of unaffected carriers of TTNtv.

The prognostic information obtained from survival curves constructed with data from the literature is concordant with the findings in our cohort and seems not to overestimate the risk of cardiovascular death in these patients. In fact, we found a trend toward a worse prognosis in our population that could be explained by the selection of the included patients.

The most frequent causes of cardiovascular death were heart failure death and heart transplantation. However, sudden deaths were also frequent, both in our cohort and in the literature.

Recent studies have observed that TTNtv carriers in non-ischemic DCM independently predicted early arrhythmias [9] and patients with TTNtv have shown an increased risk of developing ventricular arrhythmias that was independent of other covariates in patients with implanted defibrillators [10]. According to these data, DCM patients carrying TTNtv should not be considered a low arrhythmic risk group. 


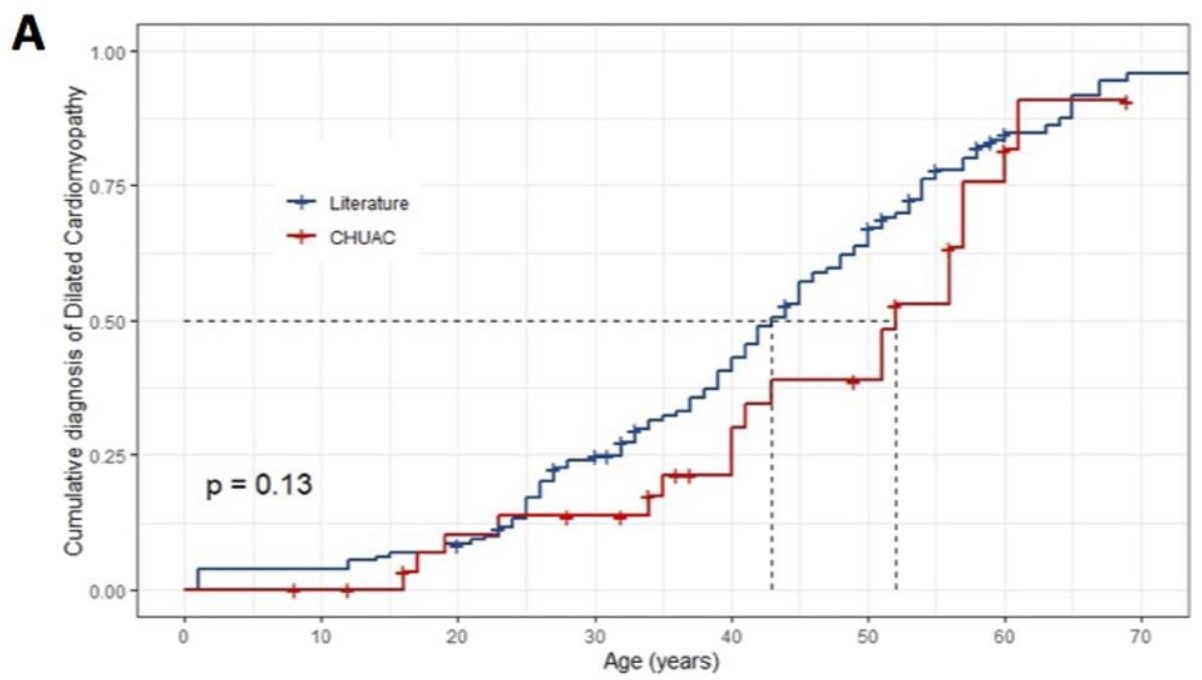

\begin{tabular}{|c|c|c|c|c|c|c|c|}
\hline 130 & 125 & 119 & 96 & 72 & 43 & 13 & 3 \\
\hline 32 & 31 & 26 & 24 & 18 & 13 & 4 & 0 \\
\hline 0 & 10 & 20 & & 40 & 50 & 60 & 70 \\
\hline
\end{tabular}
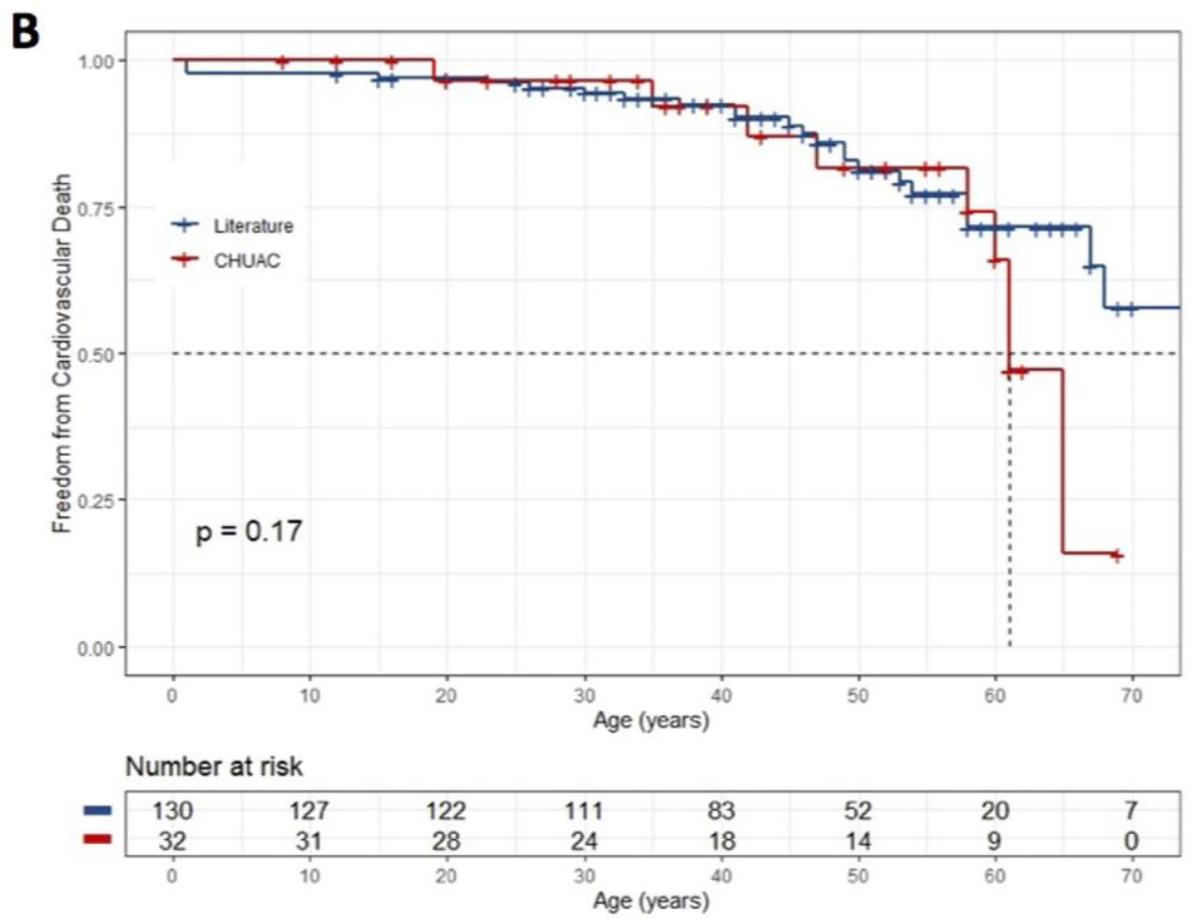

Fig. 1. Comparison between accumulated percentage of affected carriers and cardiovascular death in patients from our cohort versus patients described in the literature. 


\section{Limitations}

One of the limitations in our study was that clinical and genetic data on some of the relatives were not available and segregation could not be evaluated in several families. Also, referral and publication biases could have influenced the results toward the description of the more severe cases. In that sense, it is relevant to observe that the incidence of events in our cohort was higher than that obtained analysing data from the literature.

\section{Conclusions}

The identification of TTNtv in patients with DCM is frequent and provides relevant information about the disease prognosis. The risk of cardiovascular death and arrhythmias in these patients should not be underestimated. Age related penetrance need to be considered in the familial evaluation and follow up should be recommended in middle age unaffected carriers.

\section{Declaration of competing interest}

Drs Juan Pablo Ochoa, Marcos Cicerchia, Joel Salazar-Mendiguchia, Arsonval Lamounier, Diego Garcia-Giustiniani, Xusto Fernandez, Martin Ortiz-Genga are employees of Health in Code SL. Lorenzo Monserrat is CEO of Health in Code SL.

\section{References}

[1] T.J. Pugh, M.A. Kelly, S. Gowrisankar, et al., The landscape of genetic variation in dilated cardiomyopathy as surveyed by clinical DNA sequencing, Genet. Med. 16 (2014) 601-608.

[2] D.S. Herman, L. Lam, M.R. Taylor, et al., Truncations of titin causing dilated cardiomyopathy, N. Engl. J. Med. 366 (2012) 619-628.

[3] J.A. Jansweijer, K. Nieuwhof, F. Russo, E.T. Hoorntje, J.D. Jongbloed, R.H. Lekanne Deprez, et al., Truncating titin mutations are associated with a mild and treatable form of dilated cardiomyopathy, Eur. J. Heart Fail. 19 (2017) 512-521.

[4] M. Franaszczyk, P. Chmielewski, G. Truszkowska, et al., Titin truncating variants in dilated cardiomyopathy - prevalence and genotype-phenotype correlations, PLoS ONE 12 (2017), e0169007.

[5] A.M. Roberts, J.S. Ware, D.S. Herman, et al., Integrated allelic, transcriptional, and phenomic dissection of the cardiac effects of titin truncations in health and disease, Sci. Transl. Med. 7 (2015) 270.

[6] P. Elliott, B. Andersson, E. Arbustini, et al., Classification of the cardiomyopathies: a position statement from the European Society of Cardiology Working Group on Myocardial and Pericardial Diseases, Eur. Heart J. 29 (2008) 270-276.

[7] L. Mestroni, B. Maisch, W.J. McKenna, et al., Guidelines for the study of familial dilated cardiomyopathies. Collaborative Research Group of the European Human and Capital Mobility Project on Familial Dilated Cardiomyopathy, Eur. Heart J. 20 (1999) 93-102.

[8] S. Richards, N. Aziz, S. Bale, et al., Standards and guidelines for the interpretation of sequence variants: a joint consensus recommendation of the American College of Medical Genetics and Genomics and the Association for Molecular Pathology, Genet. Med. 17 (2015) 405-424

[9] U. Tayal, S. Newsome, R. Buchan, N. Whiffin, R. Walsh, P.J. Barton, et al., Truncating variants in titin independently predict early arrhythmias in patients with dilated cardiomyopathy, J. Am. Coll. Cardiol. 69 (2017) 2466-2468.

[10]B. Corden, J. Jarman, N. Whiffin, U. Tayal, R. Buchan, J. Sehmi, et al., Association of titintruncating genetic variants with life-threatening cardiac arrhythmias in patients with dilated cardiomyopathy and implanted defibrillators, JAMA Netw. Open 2 (2019), e196520. 